\title{
Hydrogen Liberation from Gaseous 2-Bora-1,3-diazacycloalkanium Cations
}

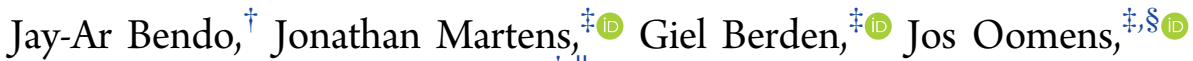 \\ and Thomas Hellman Morton ${ }^{*},+\| \odot$
}

${ }^{\dagger}$ Department of Chemistry, University of California, Riverside, 501 Big Springs Road, Riverside, California 92521 United States

${ }^{\ddagger}$ Radboud University, Institute for Molecules and Materials, FELIX Laboratory, Toernooiveld 7c, NL-6525ED, Nijmegen, The Netherlands

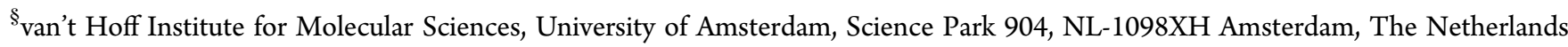

"Laboratoire de Pharmacognosie - UMR BIOCIS, Université Paris-Saclay, rue Jean-Baptiste Clément, 92296 Châtenay-Malabry, France

\section{Supporting Information}

ABSTRACT: Evidence is presented for cyclization to yield 2bora-1,3-diazacycloalkanium cations in the gas phase. While the neutral compounds in solution and solid phase are known to possess an acyclic structure (as revealed by X-ray diffraction), the gaseous cations (from which borohydride $\mathrm{BH}_{4}{ }^{-}$ion has been expelled) have a cyclic structure, as revealed by InfraRed Multiple Photon Dissociation (IRMPD) spectroscopy and collisionally activated decomposition (CAD). The IRMPD decomposition of the monocyclic ions proceeds principally via $\mathrm{H}_{2}$ expulsion, although $\mathrm{CAD}$ experiments show additional pathways. Pyrolyses of solid monomeric salts and small oligomers produce higher polymers that are consistent with $\mathrm{H}_{2}$ expulsion as the major pathway. Deuterium labeling experiments

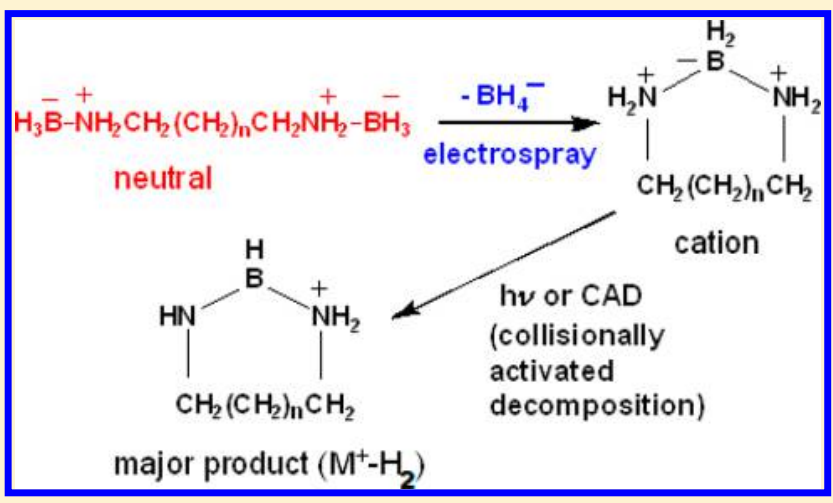
show that scrambling occurs prior to IRMPD or CAD decomposition in the gas phase.

\section{INTRODUCTION}

More than half a century ago, Kelly and Edwards reported preparation of the diamine-borane $\mathbf{1}(n=0)$ from diborane and 1,2-diaminoethane. ${ }^{1}$ At the time, they inquired whether neutral bis-adduct 1 represents the true structure, or whether it consists of an ionic solid, 2, the borohydride salt of the 5-member cyclic cation (analogous to the interconversion of ammonia borane with the diammoniate of diborane), as shown in eq $1 .^{2-6}$ They subsequently concluded that it does have the covalent structure 1,7 which was later confirmed by single crystal X-ray diffraction ${ }^{8}$ and, more recently, by Neiner et al. using X-ray powder diffraction.

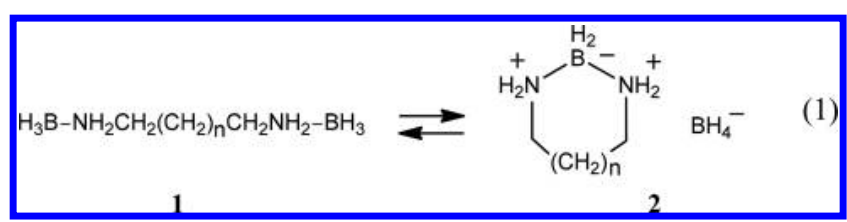

These latter investigators also report liberation of molecular hydrogen upon heating, ${ }^{9}$ with a gravimetric yield approaching $10 \%$. Subsequent workers have shown the hydrogen liberated by heating to be free from impurities. ${ }^{10}$ Attention has focused on optimizing hydrogen release, for example by exploring the anion from deprotonating $\mathbf{1}$ with sodium hydride. ${ }^{11}$ Other workers have prepared the next higher homologue of $\mathbf{1}(n=$ $1)^{12}$ in order to discover whether the hydrogen yield increases (it does not), or to incorporate $s p^{3}$ nitrogen attached to $s p^{3}$ boron in polymer chains. ${ }^{13}$

The present investigation reports spectroscopic studies on gaseous cations $(n=0-3)$ arising from electrospray mass spectrometry (ESI) of compounds 1, which suggests that the interconversion considered by Kelly and Edwards (eq 1) occurs to a measurable extent in hydroxylic solvents. One technique, IR Multiple Photon Dissociation (IRMPD) spectroscopy, isolates gaseous ions with a given $m / z$ value in a mass spectrometer and then monitors IR laser-induced decomposition as a function of wavelength. In the present case, positive ions were produced by ESI of methanolic solutions to give ions 3-12 (Chart 1) and mass-selected prior to irradiation. ${ }^{14}$ We present here studies of ions 3-6, their deuterated analogues (7-12), selected decomposition products (13-16; Chart 2), and oligomers 17-19, both by IRMPD and

Received: August 10, 2017

Revised: September 20, 2017

Published: September 22, 2017 


\section{Chart 1. Parent Ions Investigated in the Present Study}

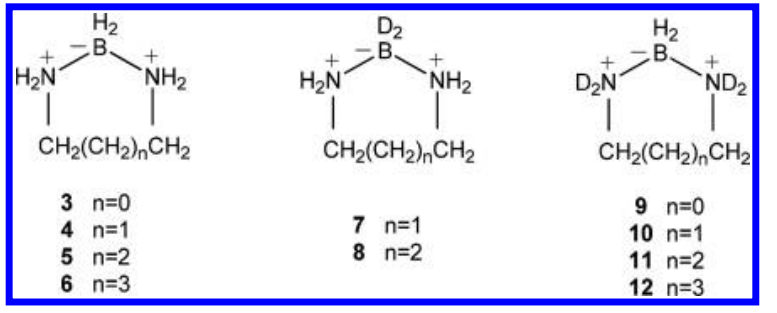

Chart 2. Principal Fragment Ions Observed in the Present Study

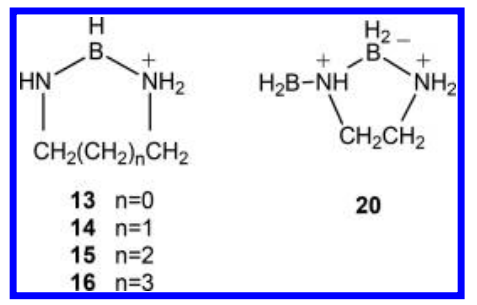

Collisionally Activated Decomposition (CAD), as well as pyrolyses of electrically neutral $\mathbf{1}(n=0$ and 1$)$, so as to probe (by mass spectrometry) the residue left by hydrogen expulsion.

The vibrational spectroscopy of the positive ions from ESI indicates the presence of cyclic cations containing $\mathrm{N}-\mathrm{B}-\mathrm{N}$ linkages among $s p^{3}$ centers. These ions and the corresponding neutral cycloalkanes are isoelectronic. Infrared (IR) spectroscopy in the gas phase reveals not only the structures of the ions but also aspects of their vibrational structure and reactivity not readily predictable from the chemistry of the isolectronic carbocycles: weak hydrogen stretching by molecular hydrogen from the gaseous ions 3-6 suggests that interconversion of 1 and the borohydride salt, 2 , as depicted in eq 1 , might play a role in producing the high yield of $\mathrm{H}_{2}$ upon decomposition.

Many years ago Shore et al. showed that the reaction of gaseous ammonia with diborane produces the diammoniate of diborane $\left(\mathrm{H}_{3} \mathrm{~N}-\mathrm{BH}_{2}-\mathrm{NH}_{3}{ }^{+} \mathrm{BH}_{4}^{-}\right)$instead of the simple Lewis acid-base adduct $\mathrm{H}_{3} \mathrm{~N}-\mathrm{BH}_{3} \cdot{ }^{2-5}$ Longer oligomers consisting of $s p^{3}$ boron bonded to $s p^{3}$ nitrogen have been synthesized, ${ }^{15,16}$ and a variety of conditions also produce other diaminoboranes containing only $s p^{3}$ centers. ${ }^{17-21}$ In the present work, reaction of linear aliphatic $\alpha, \omega$-diamines with borane yields product mixtures from which the positive ions 3-12 can be isolated by ESI mass spectrometry and their infrared spectra in the gas phase examined using the IRMPD technique.

\section{EXPERIMENTAL SECTION}

Linear diamines, borane.THF, and $2 \mathrm{M} \mathrm{BH} \cdot \mathrm{Me}_{2} \mathrm{~S}$ in THF were purchased from Acros Organics, methylene aminoacetonitrile purchased from TCI America, and $\mathrm{BD}_{3} \cdot \mathrm{THF}$ purchased from Alfa Aesar. All were used without further purification. Glassware, spin vanes, and syringes were ovendried before use. Reactions were typically performed near $0{ }^{\circ} \mathrm{C}$. In a typical experiment, an apparatus consisting of a $50 \mathrm{~mL}$ round-bottom flask fitted with a septum cap and a balloon connected via a hypodermic needle was purged in $\mathrm{N}_{2}$ while cooling from oven drying, with the balloon inflated to make sure that the apparatus remained under inert atmosphere. After the flask had cooled to room temperature, it was placed in an ice-bath, charged with diamine via syringe, and allowed to cool. Excess $\mathrm{BH}_{3} \cdot \mathrm{Me}_{2} \mathrm{~S}$ was added dropwise via a glass syringe with stirring and the solution allowed to stir in the ice-bath overnight. $\mathrm{Me}_{2} \mathrm{~S}$ and THF solvent were removed under pump vacuum, yielding a white powder, which was then dissolved in acetonitrile and passed through a silica column using ethyl acetate as eluent (approximately $100 \mathrm{~mL}$ of ethyl acetate used for every $100 \mathrm{mg}$ of crude product). Fractions were collected and solvent distilled off to give a white powder. Solutions of 36 in methanol or methanol-water are sufficiently stable for mass spectrometry to observe the positive ions resulting from ESI, but experiments conducted on acetonitrile solutions of 1 do not give any detectable gaseous cations. Dissolving 3-6 in deuterated methanol- $\mathrm{D}_{2} \mathrm{O}$ affords the deuterated ions 9-12 by ESI. For compound $\mathbf{1}(n=0)$ : IR (solid) 3229 (s), 3250 (s) [N-H symmetric and asymmetric stretches], $2977(\mathrm{~m}), 2952$ (m) [CH stretches], 2279 (s), 2335 (s) $\left[\mathrm{BH}_{3}\right.$ stretches], 1586 (s), 1571(s), 1469(s), 1338(s), 1242(s), 1200(s), 1163(s), 1112(s), 1093 (m), 1060(s), 1023(s), 940(s), 903(s), 891(s), 754(s), 698(s); ${ }^{1} \mathrm{H}$ NMR (400 MHz, $\mathrm{CD}_{3} \mathrm{CN}$ ): $\delta 3.98$ (br s, $4 \mathrm{H}, \mathrm{NH}) ; \delta 2.61(\mathrm{p}, 4 \mathrm{H}, \mathrm{CH}, \mathrm{J}=8 \mathrm{~Hz}) ; \delta 1.83(\mathrm{p}, 2 \mathrm{H}, \mathrm{CH}, \mathrm{J}=$ $8 \mathrm{~Hz}) ; \delta 1.32(\mathrm{br} \mathrm{q}, 6 \mathrm{H}, \mathrm{BH}, \mathrm{J}=92 \mathrm{~Hz})$. The assignment of the downfield methylene pentet as coming from the outer methylene groups of the alkyl chain was based on a homonuclear decoupling experiment in which irradiation of the $\mathrm{NH}$ protons collapsed that pentet to a triplet without affecting the other pentet (which is assigned to the middle methylene of the alkyl chain). The $\mathrm{BH}$ resonance appears as a 1:1:1:1 quartet from coupling to ${ }^{11} \mathrm{~B} .{ }^{11} \mathrm{~B}$ NMR $(300 \mathrm{MHz}$, $\left.\mathrm{CD}_{3} \mathrm{CN}\right)$ proton decoupled: $\delta-19.39$; coupled: $\delta-19.31\left(\mathrm{q}, \mathrm{BH}_{3}\right.$, $\mathrm{J}=297 \mathrm{~Hz}$ ). (Boron trifluoride diethyl etherate used as external reference for the ${ }^{11} \mathrm{~B}$ NMR). A small amount of the $\mathrm{B}_{3} \mathrm{H}_{8}{ }^{-}$(2$3 \%$ ) salt was detected in the ${ }^{1} \mathrm{H}$ and ${ }^{11} \mathrm{~B}$ NMR of purified samples, as well: respectively $0.14 \delta$ (dectet, $\mathrm{J}_{1_{\mathrm{H}}-11_{\mathrm{B}}}=35 \mathrm{~Hz}$ ) and $-29.39 \delta$ (unresolved), ${ }^{22}$ whose concentration diminishes upon grinding. Syntheses of the dideuterated $\left(\mathrm{BD}_{2}\right)$ derivatives of cations 7 and $\mathbf{8}$ were performed in a similar way as their undeuterated counterparts using $\mathrm{BD}_{3} \cdot \mathrm{Me}_{2} \mathrm{~S}$ in THF instead of $\mathrm{BH}_{3} \cdot$ THF. The same purification method was followed for the deuterated analogues.

Compound $1(n=0)$ was also prepared by making a solution of $3.6 \mathrm{~mL}$ concentrated sulfuric acid in $8.9 \mathrm{~mL}$ ethanol, with the ethanol solution cooled in ice-bath and the sulfuric acid added dropwise. After some stirring, methylene aminoacetonitrile was added to the solution. About $4 \mathrm{~mL}$ of additional ethanol was added to get rid of the chunks in the solution. The brownish solution was stirred for an hour at $60{ }^{\circ} \mathrm{C}$, which became yellowish and separated into two layers (white and yellow). The solution was cooled overnight and crystals formed at the interface, which was filtered off. The solid, filtered aminoacetonitrile hydrogen sulfate was dissolved in water and precipitated with ethanol twice. The free base was prepared by first dissolving the solid aminoacetonitrile hydrogen sulfate $(2.5 \mathrm{~g})$ in $2.5 \mathrm{~mL}$ methanol and $460 \mathrm{mg}$ of sodium metal was dissolved in $12 \mathrm{~mL}$ methanol to make sodium methoxide, which was added to the aminoacetonitrile hydrogen sulfate solution. The solution was stirred at room temperature for $2 \mathrm{~h}$, the resulting sodium sulfate solid filtered off, and the solvent removed to yield amino-acetonitrile as an oil, which was then dissolved in THF and reduced with 2 mol equivalents of $\mathrm{BH}_{3}$. $\mathrm{Me}_{2} \mathrm{~S}$ in THF in an ice-bath. The solution was stirred overnight, and the solvent was removed under reduced pressure to yield $\mathbf{1}$ as a white powder 
Collisionally activated decomposition (CAD) mass spectra were recorded on a Waters-Micromass Quattro Ultima triple quadrupole mass spectrometer (using argon as a collision gas) at the Service d'Analyze des Médicaments et Métabolites at the Universite Paris-Saclay.

Infrared ion spectroscopy experiments were performed at the Free Electron Laser for Infrared eXperiments (FELIX) in The Netherlands. ${ }^{23,24}$ Ions were generated via ESI and trapped in one of two tandem mass spectrometers coupled to the infrared beamlines of FELIX. In the home-built Fourier Transform Ion Cyclotron Resonance (FT-ICR) mass spectrometer, described in detail in ref 25, a Micromass Z-spray ESI source was used, and ions were accumulated for $6-15 \mathrm{~s}$ in a radio frequency $(\mathrm{rf})$ hexapole trap. The ions were pulse extracted through a quadrupole deflector and injected into the ICR cell via an rf octopole ion guide. Unwanted ions were ejected from the ICR cell by stored waveform inverse Fourier Transform (SWIFT) rf pulses applied to the excite plates of the ICR cell. Subsequently, the ions were irradiated for 5-13 s by the infrared light from the free electron laser (FEL) or from an optical parametric oscillator (OPO) system to induce IR photodissociation. At every IR frequency, 2-4 mass spectra were recorded, summed, and stored. Ions were generated from solution via ESI and guided to the QIT, where they were mass-isolated and irradiated for $0.4 \mathrm{~s}$ by the infrared light from the FEL. At every frequency step, 2-4 mass spectra were recorded, summed, and stored. Alternatively, a modified commercial Quadrupole Ion Trap (QIT, Bruker AmaZon Speed ETD) was used to record infrared photodissociation spectra of ions (see refs 23 and 24 for details). Ions were generated from solution via ESI and guided to the QIT, where they were mass-isolated and irradiated for $400 \mathrm{~ms}$ by the infrared light from the FEL. At every frequency step, 6-8 mass spectra were recorded, averaged, and stored. In the 500-1800 $\mathrm{cm}^{-1}$ range, the FELIX infrared FEL pulses have an energy of 10-60 mJ, a repetition rate of 5 or $10 \mathrm{~Hz}$, and a bandwidth of about $0.5 \%$ of the central frequency. A commercial optical parametric oscillator/amplifier (OPO/OPA) system (LaserVision, WA, USA), was used in the domain above $2000 \mathrm{~cm}^{-1}$, which delivers $5 \mathrm{~ns}$ pulses with energies ranging from $3 \mathrm{~mJ}$ at $2300 \mathrm{~cm}^{-1}$ to 20 $\mathrm{mJ}$ at $3800 \mathrm{~cm}^{-1}$ with a bandwidth of $3 \mathrm{~cm}^{-1}$ and a repetition rate of $10 \mathrm{~Hz}$. From the stored set of averaged mass spectra, an infrared spectrum can be reconstructed by plotting the normalized yield (defined as the sum of the intensities of all fragment ions divided by the sum of the intensities of all ions) as a function of IR laser frequency. The yield was corrected linearly for variations in the IR laser pulse energy.

\section{RESULTS}

Undeuterated Monocyclic Ions. Experimental ESI/ IRMPD spectra of 3-6 are reproduced in Figure 1, panels $\mathrm{B}-\mathrm{E}$, recorded using two different instruments, an ion trap (in red) and an FT-ICR (in black), using tunable infrared radiation generated by FELIX in the $500-1800 \mathrm{~cm}^{-1}$ fingerprint region. On one hand, activation of ions $3-6$ by CAD leads to expulsion of molecular hydrogen or expulsion of ammonia (along with other minor ions) to roughly comparable extents (see Supporting Information). On the other hand, activation by infrared multiple photon dissociation (IRMPD) leads principally to expulsion of hydrogen, as depicted in eq 2. Results of anharmonic DFT calculations, drawn as convolved spectra (i.e., with $20 \mathrm{~cm}^{-1}$ Gaussian peak broadening) in the top panel (A, $n$

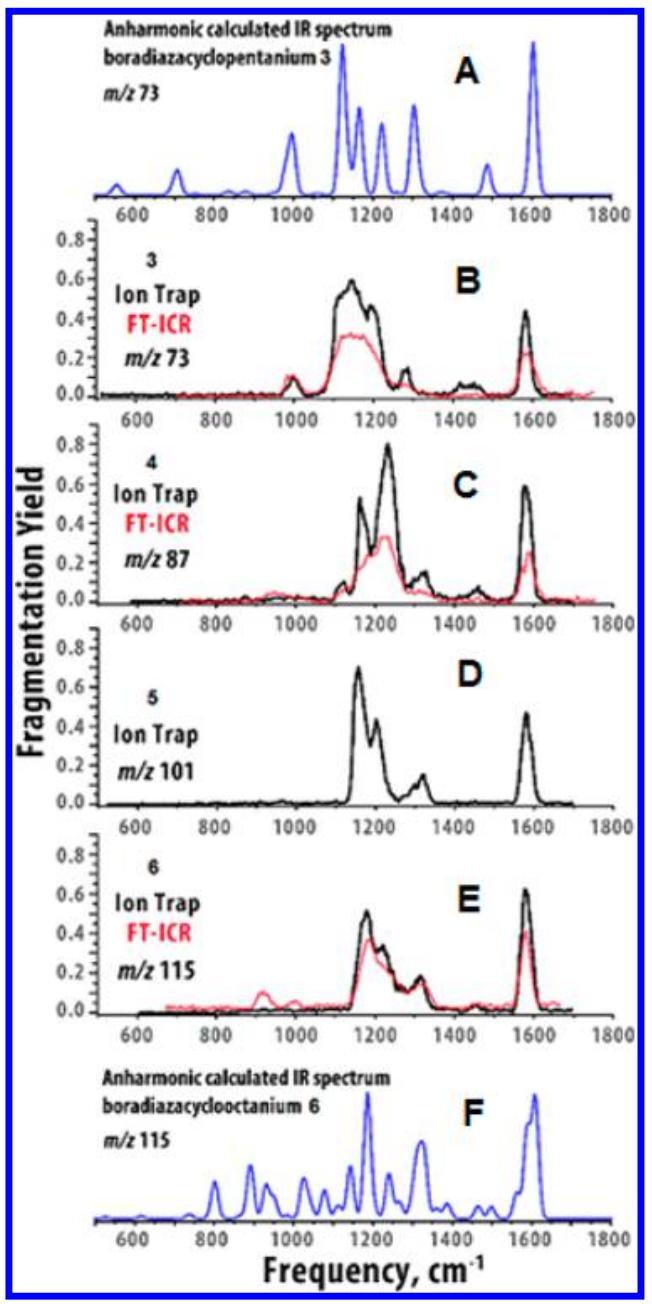

Figure 1. Calculated (B3LYP/aug-cc-pVTZ) anharmonic frequencies for 3 (top) and $\mathbf{6}$ (bottom) along with experimental gas phase IRMPD spectra in the middle (black using an ion trap, red using an FT-ICR) of 3-6. Calculated spectra have harmonic intensities convolved with 20 $\mathrm{cm}^{-1}$ Gaussian line broadening. The $y$-axis scale represents the fractional dissociation.

$=0)$ and the bottom panel $(\mathrm{F}, n=3)$, agree reasonably well with the experimental spectra in the fingerprint region.

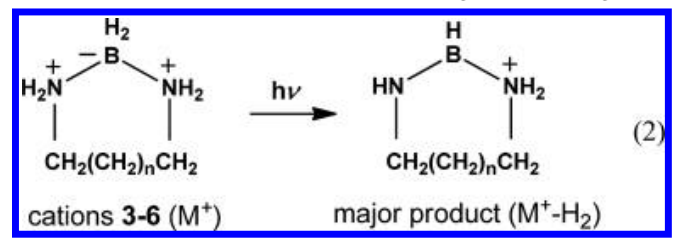

The infrared spectra obtained in the two mass spectrometers are very similar, although the ion trap IRMPD spectra show better resolution. At lower IR frequencies, the dissociation threshold is not reached for the low intensity bands in the ion trap as a consequence of the presence of helium in the QIT, which is required for efficient trapping of the ions, but which reduces the IRMPD efficiency due to collisional quenching. ${ }^{23}$ This paper describes both the IRMPD spectra and the collisionally activated decomposition (CAD) mass spectra of ring sizes 5-8 $(n=0-3)$, of several oligomers therefrom, and deuterated analogues.

Above $1100 \mathrm{~cm}^{-1}$ the spectra of the homologous series appear fairly uniform: strong bands near $1600 \mathrm{~cm}^{-1}, 1300 \mathrm{~cm}^{-1}$, and at least two bands between 1150 and $1250 \mathrm{~cm}^{-1}$, as well as 
weaker band(s) between 1400 and $1450 \mathrm{~cm}^{-1}$ (which become very weak in the 7 -membered ring $(n=2)$. Peaks appears near $1000 \mathrm{~cm}^{-1}$ in panel $\mathrm{B}(n=0)$ and at $1100 \mathrm{~cm}^{-1}$ in panel $\mathrm{C}(n=$ $1)$, which do not appear in the other spectra, although they are predicted to do so. DFT calculations predict two bands near $1600 \mathrm{~cm}^{-1}$, symmetric and antisymmetric scissors motions of the two $\mathrm{NH}_{2}$ groups, separated by only $15 \mathrm{~cm}^{-1}$ (less than the resolution of these IRMPD spectra). In the other domains, the predicted spectra experience substantial congestion, and the assignment of bands to specific vibrational modes becomes more difficult.

The IRMPD spectrum of 3 in the $\mathrm{NH}$ stretching region shows three peaks, as Figure 2 (inset C) depicts. Only two are

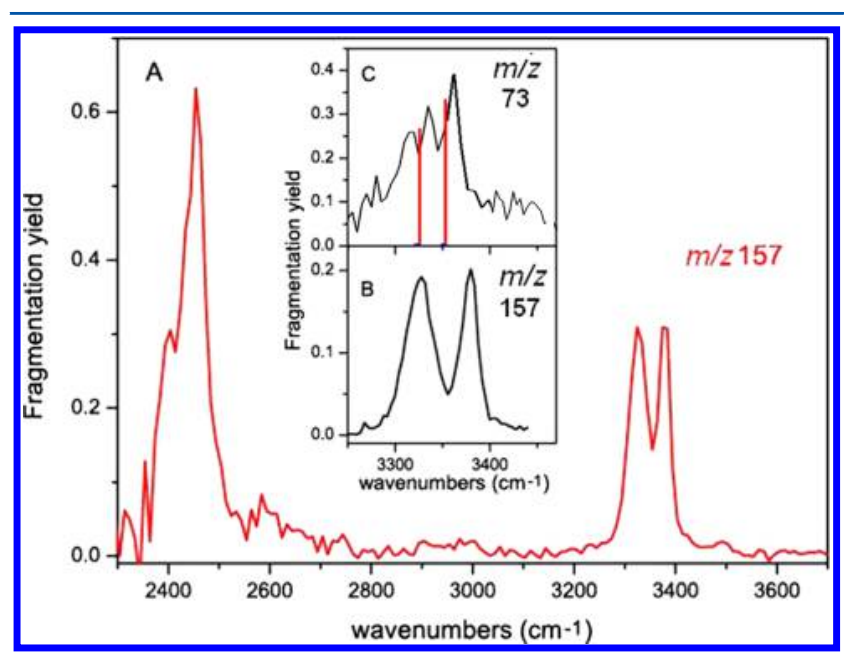

Figure 2. IRMPD spectra using an OPO laser: (A) spectrum of ion $\mathbf{1 7}$ showing $\mathrm{BH}$ stretching vibrations in the range 2435 to $2570 \mathrm{~cm}^{-1}$ (with linear correction for laser power); (B) blowup of the $\mathrm{NH}$ stretching region of 17; $(\mathrm{C})$ comparison of the experimental IRMPD spectrum of 3 (generated by $\mathrm{BH}_{3} \cdot \mathrm{Me}_{2} \mathrm{~S}$ reduction of aminoacetonitrile) with calculated line spectra (anharmonic B3LYP/aug-ccpVTZ calculation using harmonic intensities) in the $\mathrm{NH}$ stretching region.

anticipated from the calculations to be resolved (as represented by the red stick spectra) The symmetric and antisymmetric $\mathrm{NH}$ bands correspond to the stretching frequencies predicted by a DFT anharmonic calculation. The four $\mathrm{NH}$ stretches are grouped closely together in the predicted spectra, so that only two bands are expected to be resolved. Two different preparations were used, whose IRMPD spectra in this region overlap. Better resolution was achieved when the sample had been prepared by $\mathrm{BH}_{3} \cdot \mathrm{Me}_{2} \mathrm{~S}$ reduction of aminoacetonitrile (probably because the laser was better tuned), and inset $\mathrm{C}$ reproduces this spectrum.

In addition to the expulsion of $\mathrm{H}_{2}$ and ammonia (or both), other ions are observed for the larger ring sizes. For instance, $\mathrm{m} / \mathrm{z} 57$ is seen from 5 and $\mathbf{6}$ (more intensely in the IRMPD than in the CAD), which corresponds to loss of $\mathrm{H}_{2}$ and propene from 5 (or $\mathrm{H}_{2}$ and butene from 6). IRMPD of the $\mathrm{m} / z$ 57 ion from 7 confirms that ion as being $\mathrm{H}_{2} \mathrm{C}=\mathrm{NH}-\mathrm{BH}-$ $\mathrm{NH}_{2}{ }^{+}$rather than $\mathrm{H}_{2} \mathrm{C}=\mathrm{N}-\mathrm{BH}-\mathrm{NH}_{3}{ }^{+}$(spectrum in the Supporting Information). Although isotopic labeling supports that assignment, the structures of other minor ions (e.g., $m / z$ 68,70 , and $84-86$ from 6 ) cannot be reliably specified at this time.

The ions resulting from $\mathrm{H}_{2}$ loss $(13-16)$ were also examined by IRMPD. Of special interest was ion 16 , the $\mathrm{H}_{2}$-loss fragment from ion 6, because (unlike the lower homologues) the IRMPD of 6 also leads to double $\mathrm{H}_{2}$ loss in addition to single $\mathrm{H}_{2}$ loss. As can be inferred from Figure 3, the match to the scaled

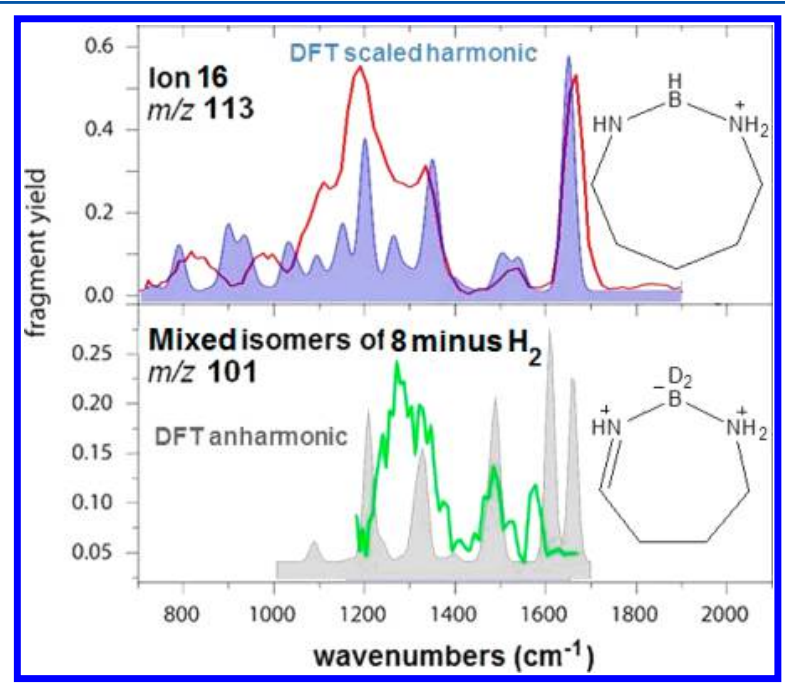

Figure 3. Top panel: IRMPD spectrum of ion 16 (red trace) compared to the scaled, calculated normal modes (silhouette) for loss of one hydrogen from boron and one from nitrogen. Bottom panel: mixture of isomers of $\mathbf{8}$ minus $\mathrm{H}_{2}$ (green trace, compared to the anharmonic spectrum shown as a silhouette) revealing evidence of isotopic scrambling prior to hydrogen expulsion. Both experimental IRMPD spectra were recorded using the FT-ICR mass spectrometer.

normal modes for the single $\mathrm{H}_{2}$-loss fragment is reasonable, suggesting that one hydrogen has been removed from boron and the other from nitrogen, ruling out a $\mathrm{C}=\mathrm{N}$ or $\mathrm{C}=\mathrm{C}$ bond or a skeletal rearrangement. As will be described below for deuterated ions, however, the loss of a second $\mathrm{H}_{2}$ does not come from boron unless isotopic transposition has occurred prior to decomposition.

Theoretically, the gas phase electronic energy differences among the smallest ring size (3), the even-numbered cycles (4 and 6 , which both have $C s$ molecular symmetry), and 5 versus their 1,2-dehydrogenated analogues $(13,14,16$, and 15, respectively), calculated at $\mathrm{B} 3 \mathrm{LYP} /$ aug-CC-pVTZ, turns out to be negligible (less than $5 \mathrm{~kJ} \mathrm{~mol}^{-1}$ difference among them). The differences among harmonic vibrational enthalpies turn out to be more substantial: $26.8 \mathrm{~kJ} \mathrm{~mol}^{-1}$ for $3 / 13$ versus $59.5 \mathrm{~kJ}$ $\mathrm{mol}^{-1}$ for $4 / 14$ and $61.3 \mathrm{~kJ} \mathrm{~mol}^{-1}$ for $6 / 16$. The isomerization of 6 to a branched structure (see Supporting Information, Figures S-8 and S-9) is exothermic by $20.1 \mathrm{~kJ} \mathrm{~mol}^{-1}$, but the barrier is apparently too high for this rearrangement to occur.

Undeuterated Oligomers. As the spectra reproduced in Figure 2 exemplify, the solid material from reaction of 1,2diaminoethane with 2 equiv of borane.THF contains not only ion $3(\mathrm{~m} / z 73)$, but also higher-order oligomers (e.g., $\mathrm{m} / z 157$ and $m / z 241$ cations), to which structures 17 and 18 are assigned in Chart 3, based on their distributions of boron isotopes, the level of agreement between DFT-computed and experimental IR bands (see Supporting Information), the observation that they exchange only 4 hydrogens with EtOD solvent (the $\mathrm{NH}_{2}$ groups but not the $\mathrm{NH}$ groups), and their fragmentations upon collisionally activated dissociation (CAD), as described below. While the OPO laser used for these experiments does not emit enough power to observe the $\mathrm{BH}$ vibrations in the IRMPD spectra of ion 3 , the IRMPD spectrum of 17 in Figure 2 (monitored by its dissociation to a mixture of 
Chart 3. Oligomerized Ions Observed from Pyrolysis of Solid Compound $1(n=0)$

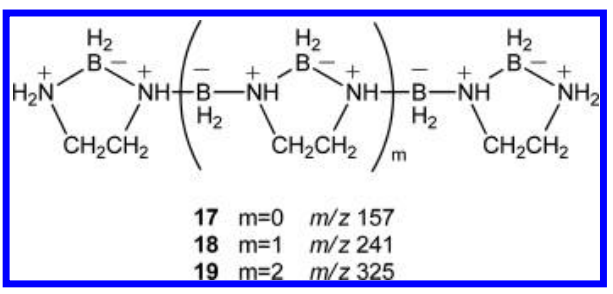

3 and 13) does show two BH stretching vibrations. Curiously, the DFT normal modes calculated for $\mathbf{1 7}$ predict a half dozen intense $\mathrm{BH}$ stretches over the domain from 2435 to $2570 \mathrm{~cm}^{-1}$ (scaled frequencies, see Supporting Information), but the experiment detects only one (or perhaps two) closely spaced bands.

$\mathrm{CAD}$ of ion $\mathbf{1 7}$ using argon gas in the middle quadrupole ( $\mathrm{rf}$ only) of a conventional triple quadrupole mass spectrometer exhibits three decomposition pathways (two of which are also seen in the IRMPD spectra): cleavage to 3, cleavage with accompanying $\mathrm{H}_{2}$ loss to 13 , and fission of one of the exocyclic $\mathrm{B}-\mathrm{N}$ bonds to yield an $\mathrm{m} / z 85$ fragment ion (which we speculate to have structure 20). Ion 18 exhibits an even simpler $\mathrm{CAD}$ pattern. The two fragment ions correspond to fission of the $\mathrm{B}-\mathrm{N}$ intersected by the left-hand parentheses in Chart 3 (to give $m / z 169$ ) or cleavage of the $\mathrm{B}-\mathrm{N}$ bond intersected by the right-hand parentheses with concomitant transfer of a boron-bound hydrogen to an expelled neutral fragment (to give $m / z 155)$. CAD of isotopomers $\left({ }^{10} \mathrm{~B}\right.$ or $d_{4}$ analogues) agree with these interpretations (see Supporting Information).

None of longer chain diamines give detectable yields of oligomers homologous to 17-19. For example, the higher mass ions from the product of 1,5-diamino- $n$-pentane with boraneTHF possess formulas of acyclic ions such as $\left[\mathrm{H}_{2} \mathrm{~N}\right.$ $\left.\left(\mathrm{CH}_{2}\right)_{5} \mathrm{NH}_{2}\right]_{m} \mathrm{BH}_{2} \mathrm{NH}_{2}\left(\mathrm{CH}_{2}\right)_{5} \mathrm{NH}_{2} \mathrm{BH}_{2}\left[\mathrm{NH}_{2}\left(\mathrm{CH}_{2}\right)_{5} \mathrm{NH}_{2}\right]_{n}$ ( $n, m=0$ or 1 , observed at $m / z 127,229$, and 331 for the ${ }^{11} \mathrm{~B}$ isotopes, respectively), instead, the heavier ones of which lose neutral 1,5-diamino-n-pentane upon CAD (see Supporting Information).

Several pyrolyses of solid $\mathbf{1}$ (both $n=0$ and $n=1$ ) were performed at $85^{\circ} \mathrm{C}$ for 1 week in sealed ampules. The goal was to examine the solid residue after $\mathrm{H}_{2}$ expulsion. Higher oligomers of 17-19 up to tetradecamers, $m=12$, as seen by ESI/ MS from the residue of $\mathbf{1}(\mathrm{n}=0)$, were observed, with increasing contributions from additional $\mathrm{H}_{2}$ loss as molecular weight increases. For example, the tetramer $(19, m / z 325)$ gives a good fit to the expected isotopic abundances expected for a pure oligomer, while the nonamer $(m=7)$ shows a better fit for an admixture containing approximately $7 \% \mathrm{H}_{2}$ loss. The Supporting Information reproduces the matches between experiment and calculations based on natural abundances.

Similarly, pyrolysis of solid $\mathbf{1}(\mathrm{n}=2)$ gives a series of oligomers in the residual solid observed up to the nonamer (which produces a sequence of peaks around $m / z 870$ ), again with increasing $\mathrm{H}_{2}$-deficiency with increasing $\mathrm{m} / z$. The admixture of $\mathrm{H}_{2}$-deficient oligomers is seen at lower molecular weights, though. For instance, the best fit for the trimer, shown in Figure $4 \mathrm{~A}$, contains an estimated $11 \%$ of an $\mathrm{H}_{2}$-deficient oligomer. IRMPD spectroscopy of the NH region of $\mathrm{m} / z 283$ (which should contain no $\mathrm{H}_{2}$-deficient product) shows a greater separation between the two observed $\mathrm{NH}$ stretching bands (approximately $80 \mathrm{~cm}^{-1}$ ) than is een in Figure 2, as well as a shift to lower frequency.

Deuterated Monocyclic lons. In order better to understand better the mechanism of $\mathrm{H}_{2}$-loss, decomposition of the series of deuterium-substituted ions 7-12 was explored. CAD of ion 9 exemplifies some consequences of deuterium labeling. Loss of $\mathrm{HD}$ and of $\mathrm{ND}_{3}$ compete to roughly equal extents, with $\mathrm{HD}$ loss $>15$ times more abundant than $\mathrm{H}_{2}$ loss. In the IRMPD experiments on $\mathbf{1 0}$ and $\mathbf{1 1}$ the major product also comes from $\mathrm{HD}$ loss, which correlates with loss of one hydrogen from boron and one from nitrogen. Figure 5 reproduces the IRMPD spectra of $7\left(d_{2}\right)$ and of $\mathbf{1 0}\left(d_{4}\right)$ and compares them with predictions from unscaled anharmonic (using harmonic intensities) and scaled harmonic frequecny calculations, respectively.

Both the harmonic (not shown) and the anharmonic frequency calculations predict that the lower frequency symmetric stretch exhibits a much less intense band than the higher frequency antisymmetric stretch. An identical result obtains for the dideuterated analogue 8 (Figure 6), which is compared with the scaled normal modes: one band is observed where two are expected. We can offer no explanation at present, except to assert that the match of the other bands clearly confirms the structures drawn.

HD loss yields the only observed dehydrogenation product from IRMPD of 7 and 10-12, but IRMPD of 8 gives nearly equal proportions of $\mathrm{H}_{2}$ and $\mathrm{HD}$ loss. Does this represent isotopic scrambling, or do two different pathways compete? To address that question, the IRMPD spectrum of the ion resulting from $\mathrm{H}_{2}$ loss $\left(8\right.$ minus $\left.\mathrm{H}_{2}\right)$ was examined. The lower panel of

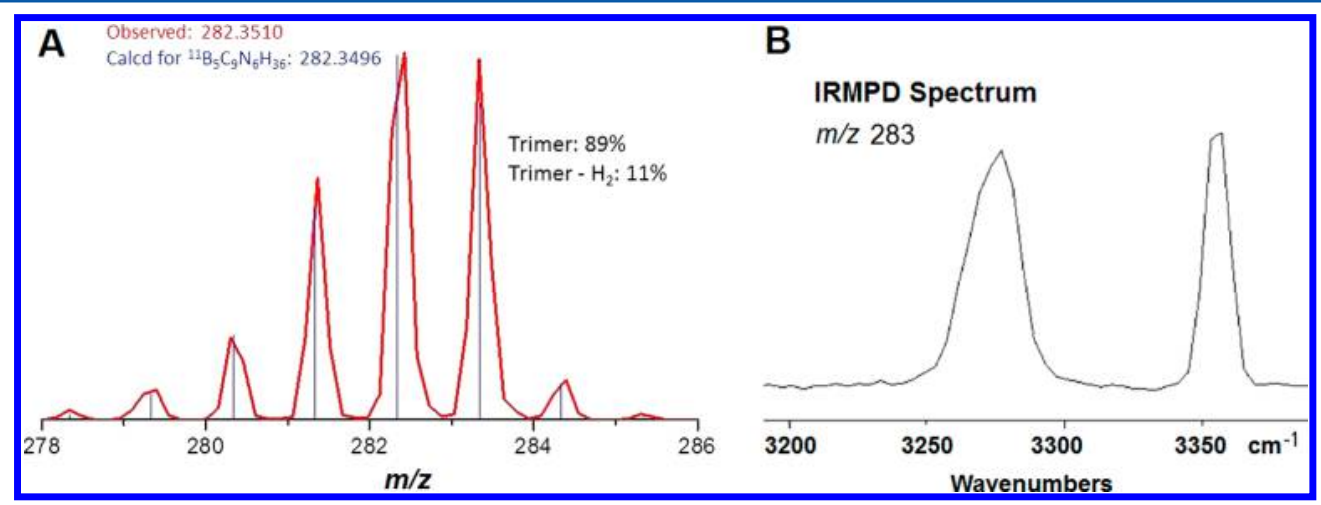

Figure 4. (A) ESI-mass spectrum of trimeric ions from pyrolysis of solid $1(n=1)$ compared to theoretical values. (B) Gas phase IRMPD spectrum in the NH stretch region of $m / z 283$ from the solid residue from pyrolysis of $\mathbf{1}(n=1)$ recorded in the FT-ICR. 


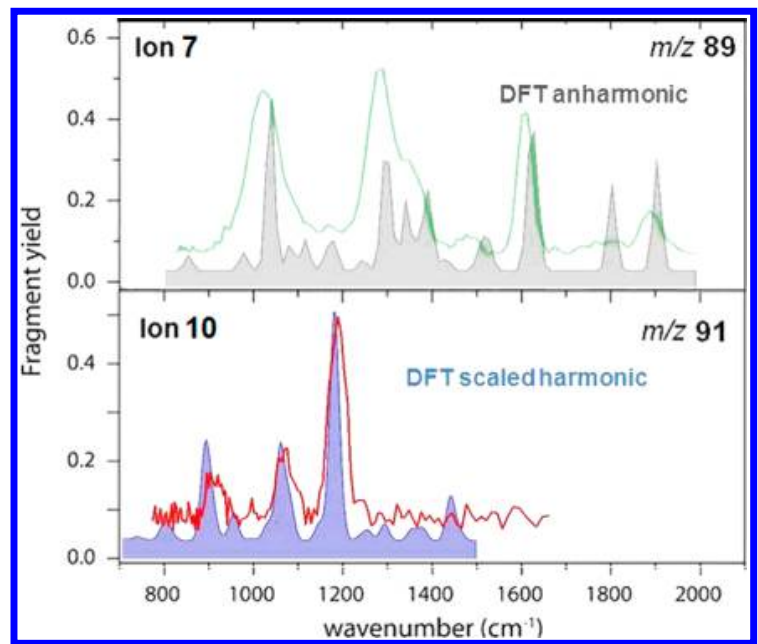

Figure 5. IRMPD spectra of deuterated analogues comparing isotopomers $7\left(d_{2}\right.$, top panel $)$ and $\mathbf{1 0}\left(d_{4}\right.$, bottom panel $)$ in the fingerprint region. The top panel compares the experimental IRMPD spectrum (green trace) with the unscaled anharmonic spectrum (silhouette), while the bottom symmetric and antisymmetric $\mathrm{BD}_{2}$ stretches for 7 ought to be well separated and have nearly the same intensity between 1800 and $1950 \mathrm{~cm}^{-1}$. However, as the top panel portrays, the panel compares the experimental IRMPD spectrum (red trace) with the scaled, calculated harmonic spectrum (silhouette). Both spectra were recorded using the FT-ICR.

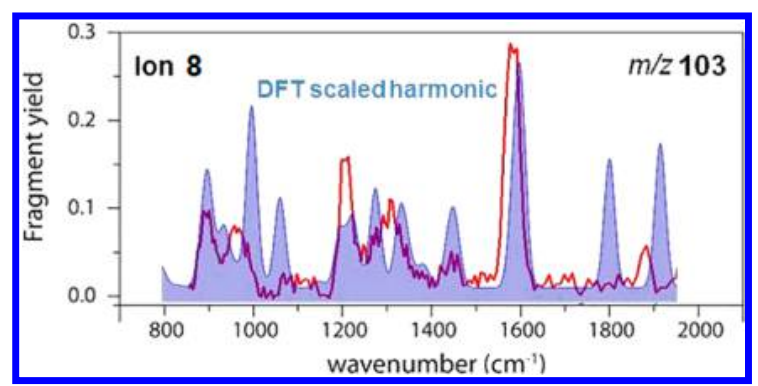

Figure 6. IRMPD spectrum of deuterated analogue $8\left(d_{2}\right.$, red trace) in the fingerprint region compared to the predicted scaled harmonic spectrum (silhouette). As in Figure 5, the symmetric and antisymmetric $\mathrm{BD}_{2}$ stretches are predicted to have the same intensity, but only the antisymmetric stretch seems to be observed by IRMPD. The spectrum was recorded using the FT-ICR.

Figure 3 compares the fingerprint region of that ion with the fingerprint region of its undeuterated higher homologue, ion 16 (upper panel). Figure 3 represents a fairly involved comparison. As mentioned above, the top panel (for the undeuterated compound) shows a good match between the predicted spectrum for 16 and the observed IRMPD spectrum. When deuterium is substituted, however, the ion resulting from $\mathrm{H}_{2}$ loss provides a very poor fit, even with regard to the unscaled anharmonic frequencies of the structure shown in the lower panel (which would result from loss of $\mathrm{H}_{2}$ from nitrogen and the adjacent carbon atom). Since the boron is completely deuterated in the precursor (ion 8), $\mathrm{H}_{2}$ loss cannot involve that atom, unless scrambling has taken place. The experimental IRMPD spectrum more resembles that of ion 15 (with deuterium substitution) with an admixture of other isomers. Hence, we infer that isotopic scrambling has occurred.

\section{DISCUSSION}

The principal band positions of the two IRMPD spectra in Figure 3 occur in the same domain. This outcome leads to the inference that deuterium transposes between boron and nitrogen prior to hydrogen expulsion, giving 8 minus $\mathrm{H}_{2}$ as a mixture of isomers. That ion is provisionally assigned to contain the four isomers of $\mathbf{1 5}$ having two deuteria partitioned between $\mathrm{N}$ and $\mathrm{B}$. In other words, the evidence supports the conclusion that each ring size 4-6 yields a single product (ions 14-16, respectively) from $\mathrm{H}_{2}$ expulsion, but that isotopic scrambling takes place upon activation prior to dissociation (at least in the case of medium sized rings).

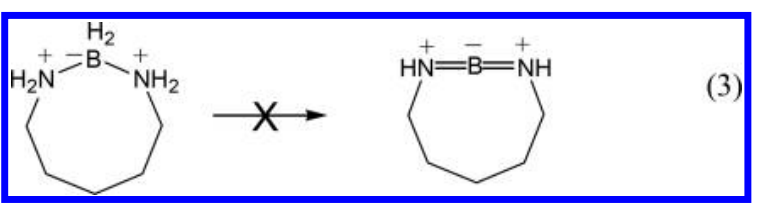

Only the 8-membered ring 6 gives rise to loss of two $\mathrm{H}_{2}$ molecules. Does this represent loss of two hydrogen atoms from nitrogen and two from boron, as eq 3 illustrates? Because the ion from double $\mathrm{H}_{2}$ loss did not have sufficient intensity for IRMPD spectroscopy, a mass spectrometric labeling experiment answers that question. Dissociation of the $d_{4}$ analogue 12 gives loss of $\mathrm{HD}$ plus $\mathrm{H}_{2}$ as the exclusive pathway for double dehydrogenation. Since the $\mathrm{N}-\mathrm{B}-\mathrm{N}$ linkage of $\mathbf{1 2}$ contains only two hydrogens (and four deuteria), the product ion must result from loss of at least one hydrogen atom from the carbon chain. Hence eq 3 does not occur to any appreciable extent (unless isotopic scrambling takes place under CAD conditions prior to decomposition).

\section{CONCLUSIONS}

Hydrogen expulsion has been observed in a homologous series of 2-bora-1,3-diazacycloalkanium cations in the gas phase, as monitored by IRMPD spectroscopy and collisionally activated decomposition ( $\mathrm{CAD})$. These ions are isoelectronic to the cycloalkanes of the same ring size. In the gas phase loss of ammonia competes with hydrogen loss, with other decomposition pathways contributing much less. Experimental evidence demonstrates that hydrogen scrambling takes place (at least in some cases) between activation and decomposition of the ions in question. Pyrolysis of solids (both monomers and oligomers) results in even higher polymers, consistent with hydrogen expulsion.

\section{ASSOCIATED CONTENT}

\section{S Supporting Information}

The Supporting Information is available free of charge on the ACS Publications website at DOI: 10.1021/acs.jpca.7b07990.

$$
\text { CAD and IRMPD spectra (19 figures) (PDF) }
$$

\section{AUTHOR INFORMATION}

\section{Corresponding Author}

*E-mail: morton@citrus.ucr.edu.

ORCID ${ }^{\circ}$

Jonathan Martens: 0000-0001-9537-4117

Giel Berden: 0000-0003-1500-922X

Jos Oomens: 0000-0002-2717-1278

Thomas Hellman Morton: 0000-0002-4122-4583 


\section{Author Contributions}

The manuscript was written through contributions of all authors. All authors have given approval to the final version of the manuscript. These authors contributed equally.

\section{Notes}

The authors declare no competing financial interest.

\section{ACKNOWLEDGMENTS}

The authors are grateful to Dr. Audrey Solgadi of SAMM at the Universite Paris-Saclay for assisting with the CAD experiments, to Erik Romero for experimental assistance, and to the FELIX staff for their skill in helping perform the IRMPD experiments (particularly Dr. A.F.G. van der Meer and Dr. B. Redlich). This work was supported, in part, by NSF grant CHE0848517 to THM. J.O. acknowledges the Nederlandse Organisatie voor Wetenschappelijk Onderzoek (NWO) for VICI grant no. 724.011.002 and thanks the Stichting Physica for support.

\section{REFERENCES}

(1) Edwards, J. O.; Kelly, H. C. Ethane 1,2-Diamineborane. I. Am. Chem. Soc. 1960, 82, 4842-4846.

(2) Schultz, D. R.; Parry, R. W. Chemical Evidence for the Structure of the "Diammoniate of Diborane." I. Evidence for the Borohydride Ion and for the Dihydro-diammineboron(III) Cation. I. Am. Chem. Soc. 1958, 80, 4-8.

(3) Shore, S. G.; Parry, R. W. Chemical Evidence for the Structure of the "Diammoniate of Diborane." II. The Preparation of AmmoniaBorane. I. Am. Chem. Soc. 1958, 80, 8-12.

(4) Parry, R. W.; Shore, S. G. Chemical Evidence for the Structure of the "Diammoniate of Diborane." III. The Reactions of Borohydride Salts with Lithium Halides and Aluminum Chloride. I. Am. Chem. Soc. 1958, 80, 12-15.

(5) Shore, S. G.; Girardot, P. R.; Parry, R. W. Chemical Evidence for the Structure of the "Diammoniate of Diborane." V. A Tracer Study of the Reaction between Sodium and the "Diammoniate of Diborane". I. Am. Chem. Soc. 1958, 80, 20-24.

(6) Chen, X.; Bao, X.; Zhao, J.-C.; Shore, S. G. Experimental and Computational Study of the Formation Mechanism of the Diammoniate of Diborane: The Role of Dihydrogen Bonds. I. Am. Chem. Soc. 2011, 133, 14172-14175.

(7) Kelly, H. C.; Edwards, J. O. Evidence for the Open Chain Structure of Ethane 1,2-Diamineborane. Inorg. Chem. 1963, 2, $226-$ 227.

(8) Ting, H. Y.; Watson, W. H.; Kelly, H. C. Molecular and Crystal Structure of Ethylenediamine-bisborane, $\mathrm{C}_{2} \mathrm{H}_{14} \mathrm{~B}_{2} \mathrm{~N}_{2}$. Inorg. Chem. 1972, 11, 374-377

(9) Neiner, D.; Karkamkar, A.; Bowden, M.; Choi, Y. J.; Luedtke, A.; Holladay, J.; Fisher, A.; Szymczak, N.; Autrey, T. Kinetic and Thermodynamic Investigation of Hydrogen Release from Ethane1,2-di-amineborane. Energy Environ. Sci. 2011, 4, 4187-4193.

(10) Leardini, F.; Valero-Pedraza, M. J.; Pérez-Mayoral; Cantelli, R.; Bañares, M. A. Thermolytic Decomposition of Ethane 1,2Diamineborane Investigated by Thermoanalytical Methods and in Situ Vibrational Spectroscopy. I. Phvs. Chem. C 2014, 118, 1722117230 .

(11) Leardini, F.; Mirabile Gattia, D.; Montone, A.; Cuevas, F.; Pérez-Mayoral, E.; Valero-Pedraza, M. J.; Bañares, M. A.; Cantelli, R. A Step Forward to the Dehydrogenation Reversibility of Amine-Borane Adducts by Coupling Sodium and Hydrocarbon Groups. Int. I. Hvdrogen Energy 2015, 40, 2763-2767.

(12) Li, L.; Gu, Q.; Tang, Z.; Chen, X.; Tan, Y.; Li, Q.; Yu, X. Two Novel Derivatives of Ammonia Borane for Hydrogen Storage: Synthesis, Structure, and Hydrogen Desorption Investigation. I. Mater. Chem. A 2013, 1, 12263-12269.

(13) Ledoux, A.; Larini, P.; Boisson, C.; Monteil, V.; Raynaud, J.; Lacôte, E. Polyboramines for Hydrogen Release: Polymers Containing
Lewis Pairs in their Backbone. Anoew. Chem. Int. Ed. 2015, 54, 15744-15749.

(14) Oomens, J.; Steill, J. D.; Morton, T. H. IR Spectra of BoronStabilized Anions in the Gas Phase. Inorg. Chem. 2010, 49, 6781-6783.

(15) Ewing, W. C.; Marchione, A.; Himmelberger, D. W.; Carroll, P. J.; Sneddon, L. G. Syntheses and Structural Characterizations of Anionic Borane-Capped Ammonia Borane Oligomers: Evidence for Ammonia Borane $\mathrm{H}_{2}$ Release via a Base-Promoted Anionic Dehydropolymerization Mechanism. I. Am. Chem. Soc. 2011, 133, 17093-17099.

(16) Ewing, W. C.; Carroll, P. J.; Sneddon, L. G. Syntheses and Characterizations of Linear Triborazanes. Inorg. Chem. 2013, 52, 10690-10697.

(17) Miller, N. E.; Muetterties, E. L. Chemistry of Boranes. XVI. A New Heterocyclic Boron Compound. Inorg. Chem. 1964, 3, 11961197.

(18) Shore, S. G.; Hickam, C. W., Jr.; Cowles, D. Unsymmetrical Cleavage of Diborane by Methylamine and Dimethylamine. I. Am. Chem. Soc. 1965, 87, 2755-2756.

(19) Haubold, W.; Schaeffer, R. Die Reaktion von Borstickstoffverbindungen mit im Lichtbogen angeregten Kohlenstoffatomen. Chem. Ber. 1971, 104, 513-518.

(20) Gragg, B. R.; Rsychkewitsch, G. E. Synthesis of a New Small Ring Heterocyclic Boron Compound. I. Am. Chem. Soc. 1974, 96, 4717-4718.

(21) Bowden, M.; Heldebrant, D. J.; Karkamkar, A.; Proffen, T.; Schenter, G. K.; Autrey, T. The Diammoniate of Diborane: Crystal Structure and Hydrogen Release. Chem. Commun. 2010, 46, 85648566.

(22) Huang, Z.; Chen, X.; Yisgedu, T.; Meyers, E. A.; Shore, S.; Zhao, J.-C. Ammonium Octahydrotriborate $\left(\mathrm{NH}_{4} \mathrm{~B}_{3} \mathrm{H}_{8}\right)$ : New Synthesis, Structure, and Hydrolytic Hydrogen Release. Inorg. Chem. 2011, 50, 3738-3742.

(23) Martens, J.; Berden, G.; Gebhardt, C. R.; Oomens, J. Infrared Ion Spectroscopy in a Modified Quadrupole Ion Trap Mass Spectrometer at the FELIX Free Electron Laser Laboratory. Rev. Sci. Instrum. 2016, 87, 103108

(24) Martens, J.; Grzetic, J.; Berden, G.; Oomens, J. Structural Identification of Electron Transfer Dissociation Products in Mass Spectrometry using Infrared Ion Spectroscopy. Nat. Commun. 2016, 7, 11754.

(25) Valle, J. J.; Eyler, J. R.; Oomens, J.; Moore, D. T.; Van der Meer, A. F. G.; Von Helden, G.; Meijer, G.; Hendrickson, C. L.; Marshall, A. G.; Blakney, G. T. Free Electron Laser- Fourier Transform Ion Cyclotron Resonance Mass Spectrometry Facility for Obtaining Infrared Multiphoton Dissociation Spectra of Gaseous Ions. Rev. Sci. Instrum. 2005, 76, 023103. 